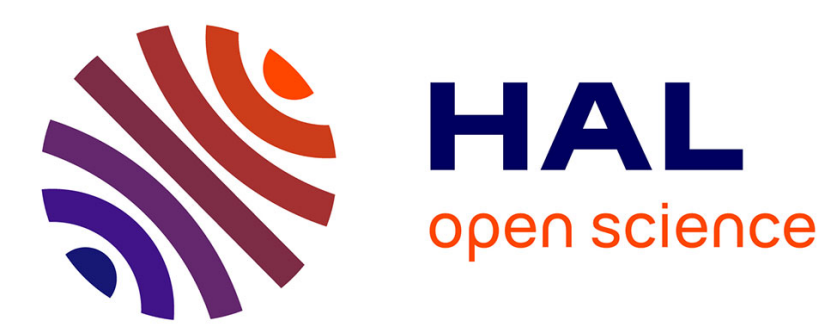

\title{
Two sided boundary stabilization of two linear hyperbolic PDEs in minimum time
}

\author{
Jean Auriol, Florent Di Meglio
}

\section{To cite this version:}

Jean Auriol, Florent Di Meglio. Two sided boundary stabilization of two linear hyperbolic PDEs in minimum time. Decision and Control (CDC), 2016 IEEE 55th Conference, Dec 2016, Las Vegas,

United States. hal-01286860

HAL Id: hal-01286860

https://hal-mines-paristech.archives-ouvertes.fr/hal-01286860

Submitted on 14 Mar 2016

HAL is a multi-disciplinary open access archive for the deposit and dissemination of scientific research documents, whether they are published or not. The documents may come from teaching and research institutions in France or abroad, or from public or private research centers.
L'archive ouverte pluridisciplinaire HAL, est destinée au dépôt et à la diffusion de documents scientifiques de niveau recherche, publiés ou non, émanant des établissements d'enseignement et de recherche français ou étrangers, des laboratoires publics ou privés. 


\title{
Two-sided boundary stabilization of two linear hyperbolic PDEs in minimum time
}

\author{
Jean Auriol ${ }^{1}$ and Florent Di Meglio ${ }^{2}$
}

\begin{abstract}
We solve the problem of stabilizing two coupled linear hyperbolic PDEs using actuation at both boundary of the spatial domain in minimum time. We design a novel Fredholm transformation similarly to backstepping approaches. This yields an explicit full-state feedback law that achieves the theoretical lower bound for convergence time to zero.
\end{abstract}

\section{INTRODUCTION}

This article solves the problem of boundary stabilization of two coupled heterodirectional linear first-order hyperbolic Partial Differential Equations (PDEs) in minimum time with one PDE in each direction and with actuation applied on both boundaries.

First-order hyperbolic PDEs are predominant in modeling of traffic flow [1], heat exchanger [26], open channel flow [9], [12] or multiphase flow [13], [14], [15]. Research on controllability and stability of hyperbolic systems have first focused on explicit computation of the solution along the characteristic curves in the framework of the $C^{1}$ norm [16], [19], [23]. Later, Control Lyapunov Functions methods emerged, enabling the design of dissipative boundary conditions for nonlinear hyperbolic systems [7], [8]. In [11] control laws for a system of two coupled nonlinear PDEs are derived, whereas in [6], [8], [21], [22], [24] sufficient conditions for exponential stability are given for various classes of quasilinear first-order hyperbolic system. These conditions typically impose restrictions on the magnitude of the coupling coefficients.

More recently, the backstepping approach has enabled the design of stabilizing full-state feedback laws. These controllers are explicit, in the sense that they are expressed as a linear functional of the distributed state at each instant. The (distributed) gains can be computed offline.

Comparing results obtained via backstepping design with existence results for stabilizing controllers reveals a gap. In [20], an extensive review of controllability results for linear hyperbolic systems is given, along with the theoretical lower bounds for convergence times. These bounds vary according, mainly, to the number and location of available actuators. Backstepping results have, until now, focused on single-boundary actuation, see e.g. [11] for the case of two coupled PDEs, [17] for an arbitrary number of PDEs or [2] for a minimum-time result in the general (single boundary actuation) case.

\footnotetext{
${ }^{1}$ Jean Auriol is with MINES ParisTech, PSL Research University, CAS - Centre automatique et systèmes, 60 bd St Michel 75006 Paris, France. jean.auriol@mines-paristech. fr

${ }^{2}$ Florent Di Meglio is with MINES ParisTech, PSL Research University, CAS - Centre automatique et systèmes, 60 bd St Michel 75006 Paris, France. florent.dimeglio@mines-paristech. fr
}

When actuation is applied at both boundaries, the literature usually focuses on design dissipative boundary conditions to stabilize the system. This not guarantee stabilization in the minimum theoretical time, and is only possible for small coupling terms between PDEs, but can generally be achieved using static boundary output feedback, which is much less computationally intensive.

In this paper, we partially bridge the gap between the existence results of [20] and the explicit control design results. More precisely, the main contribution of this paper is a minimum-time stabilizing controller in the case of two heterodirectional hyperbolic PDEs with actuation at both boundaries. A proposed boundary feedback law ensures finite-time convergence of the two states to zero in minimum time. The minimum time defined [20] is the largest time between the two transport times in each direction.

Similarly to recent approaches [5], [10], using a Fredholm transformation, the system is mapped to a target system with desirable stability properties. This target system is a copy of the original dynamics from which the coupling terms are removed. The well-posedness of the Fredholm transformation is a consequence of a clever choice of the domain on which the kernels are defined. The proof of the invertibility of this transformation is non-trivial and uses an operator-approach inspired by the one developed in [10].

The paper is organized as follow. In Section II we introduce the model equations and the notations. In Section III we present the stabilization result: the target system and its properties are presented in Section III-A. In Section III-B we derive the integral transformation and we present the domains on which the kernels are defined. Some arguments about the well-posedness of the kernels are given in Section III-C. Section IV contains the proof of the invertibility of the Fredholm transformation. In Section V we present the control feedback law and its properties. Finally in Section VI we give some simulation results.

\section{PROBlem Description}

\section{A. System under consideration}

We consider the following 2-states linear hyperbolic system

$$
\begin{aligned}
u_{t}(t, x)+\lambda u_{x}(t, x) & =\sigma^{+-} v(t, x) \\
v_{t}(t, x)-\mu v_{x}(t, x) & =\sigma^{-+} u(t, x)
\end{aligned}
$$


evolving in $\{(t, x) \mid t>0, x \in[0,1]\}$, with the following linear boundary conditions

$$
u(t, 0)=U(t), \quad v(t, 1)=V(t)
$$

with constant coupling terms and constant speeds

$$
0<\lambda \leq \mu
$$

The initial conditions denoted $u_{0}$ and $v_{0}$ are assumed to belong to $L^{2}([0,1])$.

Remark 1: The coupling terms are assumed constant here but the results of this paper can be adjusted for spatiallyvarying coupling terms.

Remark 2: System (1)-(2) is equivalent to the following system

$$
\begin{aligned}
u_{t}(t, x)+\lambda u_{x}(t, x) & =\sigma^{++} u(t, x)+\sigma^{+-} v(t, x) \\
v_{t}(t, x)-\mu v_{x}(t, x) & =\sigma^{-+} u(t, x)+\sigma^{--} v(t, x)
\end{aligned}
$$

This can straightforwardly be proved using a variable change.

Remark 3: There is no loss of generality in assuming that (4) holds.

\section{B. Well-posedness}

To study the invertibility of the Fredholm transformation used for control design, it is necessary to introduce elementary concepts of operator theory. Thus, taking the scalar product of (1)-(2) with a smooth test function $\Phi^{T}=\left(\phi_{1}, \phi_{2}\right)$ and integrating by parts leads to the following definition of a solution.

Definition 1: Consider system (1)-(2) with initial conditions $u^{0}, v^{0} \in L^{2}$ and control laws $U(t)$ and $V(t)$. We say that $\left(\begin{array}{l}u \\ v\end{array}\right)$ is a (weak) solution if for every $\tau \geq 0$ and every function $\Phi=\left(\phi_{1}, \phi_{2}\right)^{T} \in\left(C^{1}([0, \tau] \times[0,1])\right)^{2}$ such that $\phi_{1}(\cdot, 1)=\phi_{1}(\cdot, 0)=0$ we have

$$
\begin{aligned}
0= & \int_{0}^{\tau} \int_{0}^{1}-\left(\phi_{1_{t}}(t, x)+\lambda \phi_{1_{x}}(t, x)+\right. \\
& \left.\sigma^{-+} \phi_{2}(t, x)\right) u(t, x)-\left(\phi_{2_{t}}(t, x)\right. \\
& \left.-\mu \phi_{2_{x}}(t, x)+\sigma^{+-} \phi_{1}(t, x)\right) v(t, x) d x d t \\
& +\int_{0}^{1}\left(u(\tau, x) \phi_{1}(\tau, x)-u(0, x) \phi_{1}(0, x)\right. \\
& \left.+v(\tau, x) \phi_{2}(\tau, x)-v(0, x) \phi_{2}(0, x)\right) d x \\
& -\int_{0}^{\tau}\left[\lambda U(t) \phi_{1}(t, 0)+\mu V(t) \phi_{2}(t, 1)\right] d t
\end{aligned}
$$

We can consequently rewrite the system in the abstract form

$$
\frac{d}{d t}\left(\begin{array}{l}
u \\
v
\end{array}\right)=A\left(\begin{array}{l}
u \\
v
\end{array}\right)+B\left(\begin{array}{l}
U \\
V
\end{array}\right)
$$

where the operators $A$ and $B$ can be identified through their adjoints. The operator $A$ is thus defined by

$$
\begin{gathered}
A: D(A) \subset\left(L^{2}(0,1)\right)^{2} \rightarrow\left(L^{2}(0,1)\right)^{2} \\
\left(\begin{array}{l}
u \\
v
\end{array}\right) \longmapsto\left(\begin{array}{c}
-\lambda u_{x}+\sigma^{+-} v \\
\mu v_{x}+\sigma^{-+} u
\end{array}\right)
\end{gathered}
$$

with

$$
D(A)=\left\{(u, v) \in\left(H^{1}(0,1)\right)^{2} \mid u(0)=v(1)=0\right\}
$$

A is well defined and its adjoint $A^{*}$ is

$$
\begin{gathered}
A^{*}: D\left(A^{*}\right) \subset\left(L^{2}(0,1)\right)^{2} \rightarrow\left(L^{2}(0,1)\right)^{2} \\
\left(\begin{array}{l}
u \\
v
\end{array}\right) \longmapsto\left(\begin{array}{c}
\lambda u_{x}+\sigma^{-+} v \\
-\mu v_{x}+\sigma^{+-} u
\end{array}\right)
\end{gathered}
$$

with

$$
D\left(A^{*}\right)=\left\{(u, v) \in\left(H^{1}(0,1)\right)^{2} \mid u(1)=v(0)=0\right\}
$$

The operator $B$ is defined by

$$
<B\left(\begin{array}{l}
U \\
V
\end{array}\right),\left(\begin{array}{l}
z_{1} \\
z_{2}
\end{array}\right)>=\lambda U z_{1}(0)+\mu V z_{2}(1)
$$

Its adjoint is

$$
B^{*}\left(\begin{array}{l}
z_{1} \\
z_{2}
\end{array}\right)=\left(\begin{array}{l}
\lambda z_{1}(0) \\
\mu z_{2}(1)
\end{array}\right)
$$

\section{Control problem and previous results}

The goal is to design feedback control inputs $U(t)$ and $V(t)$ such that the zero equilibrium is reached in minimum time $t=t_{F}$, where

$$
t_{F}=\max \left\{\frac{1}{\mu}, \frac{1}{\lambda}\right\}=\frac{1}{\lambda}
$$

This "minimum time" is the time needed for the slowest characteristic to travel the entire length of the spatial domain. The existence of a control law reaching the null equilibrium in time $t_{F}$ is proved in [20] using a method of characteristics. To the best of our knowledge, no explicit feedback law has been designed to achieve this goal. Previous approaches yield

- exponential stability for small coupling terms when twosided static output feedback is used [3].

- finite-time stability in time $\frac{1}{\lambda}+\frac{1}{\mu}>\frac{1}{\lambda}$ when one-sided backstepping design is used, i.e with one controlled boundary only.

In the latter case, the system is mapped to a target system that has a cascade structure, which is natural for backstepping but does not enable stabilization in minimum time $t_{F}$.

\section{CONTROL DESIGN}

The control design is based on a modified backstepping approach: using a specific transformation, we map the system (1)-(3) to a target system with desirable properties of stability. However, unlike the classical backstepping approach where a Volterra transformation is used, we use a Fredholm transformation here.

\section{A. Target system design}

We map the system (1)-(3) to the following system

$$
\begin{aligned}
\alpha_{t}(t, x)+\lambda \alpha_{x}(t, x) & =\Omega(x) \beta(t, x) h_{\left[\frac{\lambda}{\lambda+\mu}, 1\right]}(x) \\
\beta_{t}(t, x)-\mu \beta_{x}(t, x) & =0
\end{aligned}
$$

with the following boundary conditions

$$
\alpha(t, 0)=0 \quad \beta(t, 1)=0
$$


$h_{I}(x)$ ( $I$ is an interval) is defined by

$$
h_{I}(x)= \begin{cases}1 & \text { if } x \in I \\ 0 & \text { else }\end{cases}
$$

while $\Omega \in L^{\infty}(0,1)$ is a function that will be defined later. This system is designed as a copy of the original dynamics, from which most of the coupling terms of (2) are removed.

Lemma 1: The zero equilibrium of (16)-(17) with boundary conditions (18) and initial conditions $\left(\alpha^{0}, \beta^{0}\right) \in$ $\mathcal{L}^{2}([0,1])$ is exponentially stable in the $\mathcal{L}^{2}$ sense.

Proof: The proof, using a Lyapunov function, is quite classical and is omitted here.

Besides, the following lemma assesses the finite-time convergence of the target system.

Lemma 2: The system (16)-(17) reaches its zero equilibrium in finite-time $t_{F}=\max \left\{\frac{1}{\lambda}, \frac{1}{\mu}\right\}=\frac{1}{\lambda}$.

Proof: Using the same arguments than the ones presented in [17, Lemma 3.1] (i.e the characteristic method), we can easily prove that for $t \geq \frac{1}{\lambda+\mu}$

$$
\begin{array}{ll}
\beta(t, x)=0 \quad \text { if } \quad x \geq \frac{\lambda}{\lambda+\mu} \\
\alpha(t, x)=0 \quad \text { if } \quad x \leq \frac{\lambda}{\lambda+\mu}
\end{array}
$$

Consequently, for $t \geq \frac{1}{\lambda+\mu}$, the system (16)-(17) can be rewritten

$$
\begin{aligned}
\alpha_{t}(t, x)+\lambda \alpha_{x}(t, x) & =0 \\
\beta_{t}(t, x)-\mu \beta_{x}(t, x) & =0
\end{aligned}
$$

with the additional conditions

$$
\alpha\left(t, \frac{\lambda}{\lambda+\mu}\right)=0 \quad \beta\left(t, \frac{\lambda}{\lambda+\mu}\right)=0
$$

Once again, using the method of characteristics, we can prove that $\forall x \in[0,1], \alpha(t, x)=0$ for $t \geq \frac{\lambda}{\lambda+\mu}+\frac{1-\frac{\lambda}{\lambda+\mu}}{\lambda}=\frac{1}{\lambda}$ and that $\beta(t, x)=0$ for $t \geq \frac{\lambda}{\lambda+\mu}+\frac{\frac{\lambda}{\lambda+\mu}}{\mu}=\frac{1}{\lambda}$.

Therefore (16)-(17) reaches its zero equilibrium in finite-time $t_{F}=\frac{1}{\lambda}$

Using an operator framework, system (16)-(17) rewrites as

$$
\frac{d}{d t}\left(\begin{array}{l}
\alpha \\
\beta
\end{array}\right)=A_{0}\left(\begin{array}{l}
\alpha \\
\beta
\end{array}\right)
$$

The operator $A_{0}$ is defined by

$$
\begin{aligned}
A_{0}: D\left(A_{0}\right) & \subset\left(L^{2}(0,1)\right)^{2} \rightarrow\left(L^{2}(0,1)\right)^{2} \\
& \left(\begin{array}{l}
\alpha \\
\beta
\end{array}\right) \longmapsto\left(\begin{array}{c}
-\lambda \alpha_{x}+\Omega \beta h_{\left[\frac{\lambda}{\lambda+\mu}, 1\right]} \\
\mu \beta_{x}
\end{array}\right)
\end{aligned}
$$

with

$$
D\left(A_{0}\right)=\left\{(\alpha, \beta) \in\left(H^{1}(0,1)\right)^{2} \mid \alpha(0)=\beta(1)=0\right\}
$$

$A_{0}$ is well defined and its adjoint $A_{0}^{*}$ is

$$
\begin{aligned}
A_{0}^{*}: D\left(A_{0}^{*}\right) & \subset\left(L^{2}(0,1)\right)^{2} \rightarrow\left(L^{2}(0,1)\right)^{2} \\
& \left(\begin{array}{c}
\alpha \\
\beta
\end{array}\right) \longmapsto\left(\begin{array}{c}
\lambda \alpha_{x} \\
-\mu \beta_{x}+\Omega \alpha h_{\left[\frac{\lambda}{\lambda+\mu}, 1\right]}
\end{array}\right)
\end{aligned}
$$

with

$$
D\left(A_{0}^{*}\right)=\left\{(\alpha, \beta) \in\left(H^{1}(0,1)\right)^{2} \mid \alpha(1)=\beta(0)=0\right\}
$$

\section{B. Fredholm transformation}

1) Definition of the transformation: Without any loss of generality we recall that $\lambda \leq \mu$. In order to map the original system (1)-(3) to the target system (16)-(18), we use the following transformation

$$
\begin{aligned}
& \alpha(t, x)=u(t, x) \\
& +h_{\left[0, \frac{\lambda}{\mu+\lambda}\right]}(x) \int_{x}^{-\frac{\mu}{\lambda} x+1}(K(x, \xi) u(t, \xi)+L(x, \xi) v(t, \xi)) d \xi \\
& +h_{]^{\frac{\lambda}{\mu+\lambda}}, 1\right]}(x) \int_{\frac{\lambda}{\mu}(1-x)}^{x}(M(x, \xi) u(t, \xi)+N(x, \xi) v(t, \xi)) d \xi \\
& \beta(t, x)=v(t, x) \\
& +h_{\left[0, \frac{\lambda}{\mu+\lambda}\right]}(x) \int_{x}^{\frac{\lambda}{\mu}(1-x)}(\bar{K}(x, \xi) u(t, \xi)+\bar{L}(x, \xi) v(t, \xi)) d \xi \\
& +h_{]_{\frac{\lambda}{\mu+\lambda}}, 1\right]}(x) \int_{\frac{\lambda}{\mu}(1-x)}^{x}(\bar{M}(x, \xi) u(t, \xi)+\bar{N}(x, \xi) v(t, \xi)) d \xi
\end{aligned}
$$

where, for any interval I, $h_{I}(x)$ is defined by

$$
h_{I}(x)= \begin{cases}1 & \text { if } x \in I \\ 0 & \text { else }\end{cases}
$$

We define the following triangular domains, depicted in Figure 1 and Figure 2:

$$
\begin{aligned}
& \mathcal{T}_{0}=\left\{(x, \xi) \mid \quad x \in\left[0, \frac{\lambda}{\lambda+\mu}\right], \quad x \leq \xi \leq \frac{\mu}{\lambda}(1-x)\right\} \\
& \overline{\mathcal{T}}_{0}=\left\{(x, \xi) \mid \quad x \in\left[0, \frac{\lambda}{\lambda+\mu}\right], \quad x \leq \xi<\frac{\lambda}{\mu}(1-x)\right\} \\
& \left.\left.\overline{\mathcal{T}}_{1}=\{(x, \xi) \mid \quad x \in] \frac{\lambda}{\lambda+\mu}, 1\right], \quad \frac{\lambda}{\mu}(1-x)<\xi \leq x\right\}
\end{aligned}
$$

The kernels $K, L$ are defined on $\mathcal{T}_{0}, M, N$ are defined on $\overline{\mathcal{T}}_{1}$. The kernels $\bar{K}, \bar{L}$ are defined on $\overline{\mathcal{T}}_{0}$ and $\bar{M}, \bar{N}$ are defined on $\overline{\mathcal{T}}_{1}$. They are continuous in their domains of assumed definition. They all have yet to be defined.

Remark 4: One may think that due to the use of the $h$ functions, the transformation presents a discontinuity in $x=$ $\frac{\lambda}{\mu+\lambda}$. Nevertheless, one can check that the right and left limits are equal since the integral vanishes and that consequently we do not have any discontinuity.

Remark 5: This transformation is a Fredholm transformation and can be rewritten using integrals between 0 and 1 as follows

$\alpha(t, x)=u(t, x)-\int_{0}^{1} Q_{11}(x, \xi) u(t, \xi)+Q_{12}(x, \xi) v(t, \xi) d \xi$

$\beta(t, x)=v(t, x)-\int_{0}^{1} Q_{21}(x, \xi) u(t, \xi)+Q_{22}(x, \xi) v(t, \xi) d \xi$ 
with

$$
\begin{aligned}
Q_{11}(x, \xi) & =-K(x, \xi) h_{\left[x,-\frac{\mu}{\lambda} x+1\right]}(\xi) h_{\left[0, \frac{\lambda}{\lambda+\mu}\right]}(x) \\
& -M(x, \xi) h_{\left[\frac{\lambda}{\mu}(1-x), x\right]}(\xi) h_{]_{\frac{\lambda}{\lambda+\mu}}, 1\right]}(x) \\
Q_{12}(x, \xi) & =-L(x, \xi) h_{\left[x,-\frac{\mu}{\lambda} x+1\right]}(\xi) h_{\left[0, \frac{\lambda}{\lambda+\mu}\right]}(x) \\
& -N(x, \xi) h_{\left[\frac{\lambda}{\lambda}(1-x), x\right]}(\xi) h_{]_{\frac{\lambda}{\lambda+\mu}}, 1\right]}(x) \\
Q_{21}(x, \xi) & =-\bar{K}(x, \xi) h_{\left[x, \frac{\lambda}{\mu}(1-x)\right]}(\xi) h_{\left[0, \frac{\lambda}{\lambda+\mu}\right]}(x) \\
& -\bar{M}(x, \xi) h_{\left[\frac{\lambda}{\mu}(1-x), x\right]}(\xi) h_{]_{\left.\frac{\lambda}{\lambda+\mu}, 1\right]}}(x) \\
Q_{22}(x, \xi) & =-\bar{L}(x, \xi) h_{\left[x, \frac{\lambda}{\mu}(1-x)\right]}(\xi) h_{\left[0, \frac{\lambda}{\lambda+\mu}\right]}(x) \\
& -\bar{N}(x, \xi) h_{\left[\frac{\lambda}{\mu}(1-x), x\right]}(\xi) h_{]_{\left.\frac{\lambda}{\lambda+\mu}, 1\right]}(x)}(x)
\end{aligned}
$$

Remark 6: Since $\alpha(0)=\beta(1)=0$ the two control laws $U$ and $V$ can be computed as functions of $(u, v)$.

2) Kernel equations: We now differentiate the Fredholm transformation (30)-(31) with respect to time and space to compute the equations satisfied by the kernels. We start with the $\beta$-transformation (31)

if $x \geq \frac{\lambda}{\mu+\lambda}$ : Differentiating (31) with respect to space and using the Leibniz rule yields

$$
\begin{aligned}
\beta_{x}(t, x)= & v_{x}(t, x)+\bar{M}(x, x) u(t, x)+\bar{N}(x, x) v(t, x) \\
& +\frac{\lambda}{\mu} \bar{M}\left(x, \frac{\lambda}{\mu}(1-x)\right) u\left(t, \frac{\lambda}{\mu}(1-x)\right) \\
& +\frac{\lambda}{\mu} \bar{N}\left(x, \frac{\lambda}{\mu}(1-x)\right) v\left(t, \frac{\lambda}{\mu}(1-x)\right) \\
& +\int_{\frac{\lambda}{\mu}(1-x)}^{x} \bar{M}_{x}(x, \xi) u(t, \xi)+\bar{N}_{x}(x, \xi) v(t, \xi) d \xi
\end{aligned}
$$

Differentiating (31) with respect to time, using (1), (2) and integrating by parts yields

$$
\begin{aligned}
& \beta_{t}(t, x)=\mu v_{x}(t, x)+\sigma^{-+} u(t, x)+ \\
& \mu \bar{N}(x, x) v(t, x)-\mu \bar{N}\left(x, \frac{\lambda}{\mu}(1-x)\right) v\left(t, \frac{\lambda}{\mu}(1-x)\right) \\
& -\lambda \bar{M}(x, x) u(t, x)+\lambda \bar{M}\left(x, \frac{\lambda}{\mu}(1-x)\right) u\left(t, \frac{\lambda}{\mu}(1-x)\right) \\
& \quad+\int_{\frac{\lambda}{\mu}(1-x)}^{x} \lambda \bar{M}_{\xi}(x, \xi) u(t, \xi)-\mu \bar{N}_{\xi}(x, \xi) v(t, \xi) \\
& \quad+\sigma^{-+} \bar{N}(x, \xi) u(t, \xi)+\sigma^{+-} \bar{M}(x, \xi) v(t, \xi) d \xi
\end{aligned}
$$

Plugging these expressions into the target system (16)-(17) yields the following system of kernel equations

$$
\begin{aligned}
0 & =-\mu \bar{M}_{x}(x, \xi)+\lambda \bar{M}_{\xi}(x, \xi)+\sigma^{-+} \bar{N}(x, \xi) \\
0 & =-\mu \bar{N}_{x}(x, \xi)-\mu \bar{N}_{\xi}(x, \xi)+\sigma^{+-} \bar{M}(x, \xi) \\
0 & =\bar{M}(x, x)-\frac{\sigma^{-+}}{\lambda+\mu} \\
0 & =\bar{N}\left(x, \frac{\lambda}{\mu}(1-x)\right)
\end{aligned}
$$

if $x<\frac{\lambda}{\mu+\lambda}$ : Similarly we get

$$
\begin{aligned}
0 & =-\mu \bar{K}_{x}(x, \xi)+\lambda \bar{K}_{\xi}(x, \xi)+\sigma^{-+} \bar{L}(x, \xi) \\
0 & =-\mu \bar{L}_{x}(x, \xi)-\mu \bar{L}_{\xi}(x, \xi)+\sigma^{+-} \bar{K}(x, \xi) \\
0 & =\bar{K}(x, x)+\frac{\sigma^{-+}}{\lambda+\mu} \\
0 & =\bar{L}\left(x, \frac{\lambda}{\mu}(1-x)\right)
\end{aligned}
$$

The corresponding domains, characteristic lines and boundary conditions in Figure 1 We now focus on the alpha-

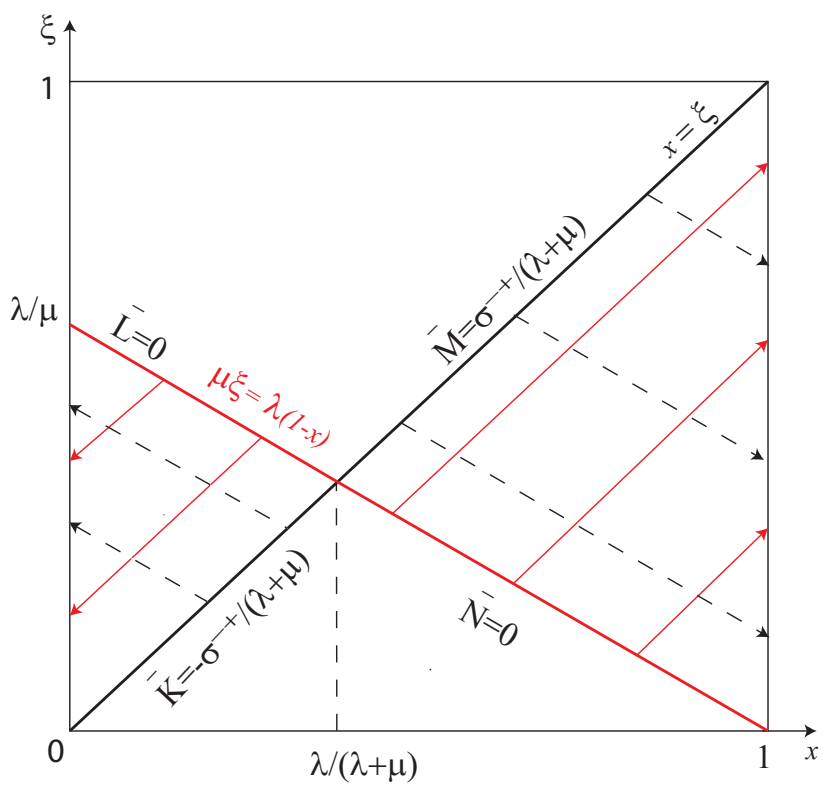

Fig. 1. Representation of the beta-kernels

transformation.

if $x \leq \frac{\lambda}{\mu+\lambda}$ : As above, differentiating (30) with respect to space and time and then plugging into the target system (16)(17) yields the following system of kernel equations

$$
\begin{aligned}
& 0=\lambda L_{x}(x, \xi)-\mu L_{\xi}(x, \xi)+\sigma^{+-} K(x, \xi) \\
& 0=\lambda K_{x}(x, \xi)+\lambda K_{\xi}(x, \xi)+\sigma^{-+} L(x, \xi) \\
& 0=L(x, x)-\frac{\sigma^{+-}}{\lambda+\mu} \\
& 0=K\left(x, \frac{\mu}{\lambda}(1-x)\right)
\end{aligned}
$$


if $x>\frac{\lambda}{\mu+\lambda}$ : Similarly we get

$$
\begin{aligned}
0 & =\lambda M_{x}(x, \xi)+\lambda \bar{M}_{\xi}(x, \xi)+\sigma^{-+} N(x, \xi) \\
& -(\lambda+\mu) \bar{M}(x, \xi) N(x, x)-\sigma^{+-} \bar{M}(x, \xi) \\
0 & =\lambda N_{x}(x, \xi)-\mu N_{\xi}(x, \xi)+\sigma^{+-} M(x, \xi) \\
& -(\lambda+\mu) \bar{N}(x, \xi) N(x, x)-\sigma^{+-} \bar{N}(x, \xi) \\
0 & =N\left(x, \frac{\lambda}{\mu}(1-x)\right) \\
0 & =M\left(x, \frac{\lambda}{\mu}(1-x)\right)
\end{aligned}
$$

In order to have a well-posed system, we add the following artificial boundary condition

$$
N(1, \xi)=0
$$

The function $\Omega(x)$ is defined by

$$
\Omega(x)=\sigma^{+-}+(\mu+\lambda) N(x, x)
$$

The corresponding domains, characteristic lines and boundary conditions in Figure 2.

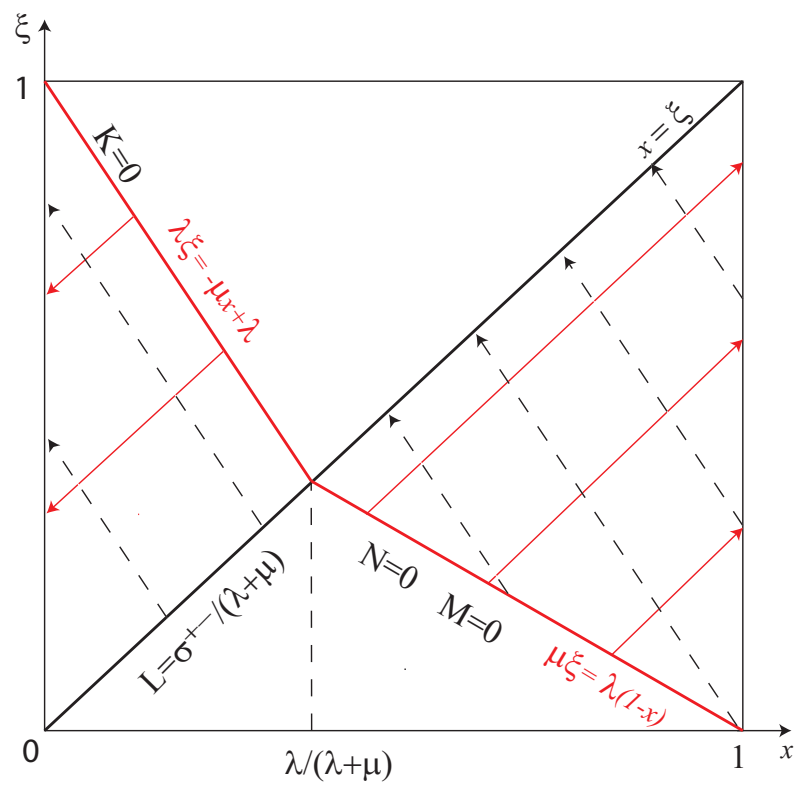

Fig. 2. Representation of the alpha-kernels

Remark 7: The artificial boundary condition we add for the kernel $N$ is not a degree of freedom since it has no impact on the control law and on the stability of the target system.

\section{Well-posedness of the kernel equations}

Theorem 1: Consider systems (44)-(47), (48)-(51), (52)(55), (56)-(60). There exists a unique solution $K, L$ (defined on $L^{\infty}(\mathcal{T})$ ), $M, N$ (defined on $\left.L^{\infty}\left(\overline{\mathcal{T}}_{1}\right)\right), \bar{K}, \bar{L}$ (defined on $\left.L^{\infty}\left(\overline{\mathcal{T}}_{0}\right)\right), \bar{M}, \bar{N}$ (defined on $L^{\infty}\left(\overline{\mathcal{T}}_{1}\right)$ ).

Classically (see [17], [18] and [25]) the proof of this theorem consists in transforming the kernel equations into integral equations using the method of the characteristics. These integral equations are then solved using the method of successive approximations. We start with the systems (44)(47), (48)-(51),(52)-(55) and finish with the system (56)-(60) since for this last one we need to use the fact that $\bar{M}(x, \xi)$ and $\bar{N}(x, \xi)$ are bounded.

\section{INVERTIBILITY OF THE FREDHOLM TRANSFORMATION}

Unlike the Volterra transformation, the Fredholm transformation is not always invertible. In [10], the authors prove the invertibility of such a transformation in the case of a first-order integro-differential hyperbolic equation. In this section we use similar arguments (in particular we rely on the Fredholm alternative) to prove the invertibility of our transformation.

1) Operator formulation of the Fredholm transformation and properties: In this subsection we rewrite the previous Fredholm transformation using operators. This will lead to some relations verified by the adjoint operators. The Fredholm transformation (36)-(37) can be written as an operator $P$ acting on $\left(\begin{array}{l}u \\ v\end{array}\right)$. More precisely we have

$$
\begin{gathered}
P=I d_{2}-Q \\
\left(\begin{array}{l}
\alpha \\
\beta
\end{array}\right)=P\left(\begin{array}{l}
u \\
v
\end{array}\right)
\end{gathered}
$$

where $Q:\left(L^{2}(0,1)\right)^{2} \rightarrow\left(L^{2}(0,1)\right)^{2}$ is the integral operator defined by

$$
Q\left(\begin{array}{l}
u \\
v
\end{array}\right)=\int_{0}^{1}\left(\begin{array}{l}
Q_{11}(x, \xi) u(t, \xi)+Q_{12}(x, \xi) v(t, \xi) \\
Q_{21}(x, \xi) u(t, \xi)+Q_{22}(x, \xi) v(t, \xi)
\end{array}\right) d \xi
$$

Its adjoint is:

$$
Q^{*}\left(\begin{array}{l}
u \\
v
\end{array}\right)=\int_{0}^{1}\left(\begin{array}{l}
Q_{11}(\xi, x) u(t, \xi)+Q_{21}(\xi, x) v(t, \xi) \\
Q_{12}(\xi, x) u(t, \xi)+Q_{22}(\xi, x) v(t, \xi)
\end{array}\right) d \xi
$$

One can easily check that:

$$
Q^{*}\left(D\left(A^{*}\right)\right) \subset D\left(A^{*}\right)
$$

The control $\left(\begin{array}{l}U \\ V\end{array}\right)$ can also be rewritten using operators

$$
\left(\begin{array}{l}
U \\
V
\end{array}\right)=\Gamma\left(\begin{array}{l}
u \\
v
\end{array}\right)
$$

with

$$
\Gamma\left(\begin{array}{l}
u \\
v
\end{array}\right)=\int_{0}^{1}\left(\begin{array}{l}
Q_{11}(0, \xi) u(t, \xi)+Q_{12}(0, \xi) v(t, \xi) \\
Q_{21}(1, \xi) u(t, \xi)+Q_{22}(1, \xi) v(t, \xi)
\end{array}\right) d \xi
$$


Using (25) and (63) yields

$$
\frac{d}{d t}\left(\begin{array}{l}
\alpha \\
\beta
\end{array}\right)=A_{0}\left(\begin{array}{l}
\alpha \\
\beta
\end{array}\right)=A_{0} P\left(\begin{array}{l}
u \\
v
\end{array}\right)
$$

Moreover using (8) and (63) we get

$$
\begin{aligned}
\frac{d}{d t}\left(\begin{array}{l}
\alpha \\
\beta
\end{array}\right) & =\frac{d}{d t}\left(P\left(\begin{array}{l}
u \\
v
\end{array}\right)\right) \\
& =P A\left(\begin{array}{l}
u \\
v
\end{array}\right)+P B \Gamma\left(\begin{array}{l}
u \\
v
\end{array}\right)
\end{aligned}
$$

Consequently $P$ and $\Gamma$ satisfy the following relation:

$$
A_{0} P=P A+P B \Gamma
$$

Taking the adjoints, this is equivalent to

$$
P^{*} A_{0}^{*}=A^{*} P^{*}+\Gamma^{*} B^{*} P^{*}
$$

2) The Fredholm alternative: We give first the following useful lemmas:

Lemma 3: $\operatorname{ker} P^{*} \subset D\left(A_{0}^{*}\right)=D\left(A^{*}\right)$

Proof: Let us consider $z \in \operatorname{ker} P^{*}$. Consequently we have $P^{*} z=0$. We can rewrite it

$$
\left(\begin{array}{l}
z_{1} \\
z_{2}
\end{array}\right)=\int_{0}^{1}\left(\begin{array}{l}
Q_{11}(\xi, x) z_{1}(t, \xi)+Q_{21}(\xi, x) z_{2}(t, \xi) \\
Q_{12}(\xi, x) z_{1}(t, \xi)+Q_{22}(\xi, x) z_{2}(t, \xi)
\end{array}\right) d \xi
$$

If we evaluate the first line for $x=1$ and the second one for $x=0$, using the fact that $Q_{11}(\xi, 0)=Q_{21}(\xi, 0)=$ $Q_{12}(\xi, 1)=Q_{22}(\xi, 1)=0$, we get

$$
z_{1}(1)=z_{2}(0)=0
$$

Consequently $z \in D\left(A_{0}^{*}\right)$ and we can write

$$
\operatorname{ker} P^{*} \subset D\left(A_{0}^{*}\right)
$$

\section{Lemma 4: $\operatorname{ker} P^{*} \subset \operatorname{ker} B^{*}$}

Proof: Let us consider $z \in \operatorname{ker} P^{*}$. Consequently we have $P^{*} z=0$. We can rewrite it

$$
\left(\begin{array}{l}
z_{1} \\
z_{2}
\end{array}\right)=\int_{0}^{1}\left(\begin{array}{l}
Q_{11}(\xi, x) z_{1}(t, \xi)+Q_{21}(\xi, x) z_{2}(t, \xi) \\
Q_{12}(\xi, x) z_{1}(t, \xi)+Q_{22}(\xi, x) z_{2}(t, \xi)
\end{array}\right) d \xi
$$

If we evaluate the first line for $x=0$ and the second one for $x=1$, using the fact that $Q_{11}(\xi, 1)=Q_{21}(\xi, 1)=$ $Q_{12}(\xi, 0)=Q_{22}(\xi, 0)=0$, we get

$$
z_{1}(0)=z_{2}(1)=0
$$

Consequently $z \in \operatorname{ker} B^{*}$ and we can write

$$
\operatorname{ker} P^{*} \subset \operatorname{ker} B^{*}
$$

Lemma 5: $\forall \lambda \in \Re \operatorname{ker}\left(\lambda I d_{2}-A_{0}^{*}\right) \cap \operatorname{ker} B^{*}=\{0\}$

Proof: Let us consider $\nu \in \Re$ and $z \in \operatorname{ker}\left(\nu I d_{2}-A_{0}^{*}\right) \cap$ ker $B^{*}=\{0\}$. Consequently we have

$$
\left(\begin{array}{c}
\lambda z_{1_{x}}(t, x)-\nu z_{1}(t, x) \\
-\mu z_{2_{x}}(t, x)-\nu z_{2}(t, x)
\end{array}\right)=\left(\begin{array}{l}
0 \\
0
\end{array}\right)
$$

with the boundary conditions

$$
z_{1}(0)=z_{2}(0)=0
$$

Consequently we have $z=\left(\begin{array}{l}0 \\ 0\end{array}\right)$

We can now state the following theorem

Theorem 2: The map $P^{*}=I d_{2}-Q^{*}$ is invertible

Proof: Since $Q^{*}$ is a compact operator we can use the Fredholm alternative (e.g [4]): $I d_{2}-Q^{*}$ is either noninjective or surjective. Consequently it suffices to prove that $P^{*}$ is injective. In addition, the Fredholm alternative also gives [4]

$$
\operatorname{dim} \operatorname{ker}\left(I d-Q^{*}\right)<+\infty
$$

By contradiction we assume that ker $P^{*} \neq\{0\}$. We first prove that ker $P^{*}$ is stable by $A_{0}^{*}$. We have ker $P^{*} \subset D\left(A_{0}^{*}\right)$. Let then consider $z \in \operatorname{ker} P^{*}$. Using (72) we can obtain

$$
P^{*} A_{0}^{*} z=0
$$

We thus have $A_{0}^{*} z \in \operatorname{ker} P^{*}$. Consequently the restriction $A_{0_{\mid \mathrm{ker} P^{*}}}$ of $A_{0}^{*}$ to $\operatorname{ker} P^{*}$ is a linear operator from $\operatorname{ker} P^{*}$ to $\operatorname{ker} P^{*}$. Since the dimension of ker $P^{*}$ is finite we can find at least one eigenvalue $\nu$. Let $e \in \operatorname{ker} P^{*}$ be a corresponding eigenvector (by definition $e \neq 0$ ). We have $e \in \operatorname{ker} P^{*}$ and so $e \in \operatorname{ker} B^{*}$. Moreover we have $A_{0}^{*} e=\nu e$. Consequently

$$
e \in \operatorname{ker}\left(\nu I d_{2}-A_{0}^{*}\right) \cap \operatorname{ker} B^{*}
$$

which contradicts Lemma 5 and concludes the proof.

\section{CONTROL LAW AND MAIN RESUlts}

We now state the main stabilization result as follows:

Theorem 3: System (1)-(2) with the following feedback control laws

$$
\begin{gathered}
U(t)=-\int_{0}^{1}(K(0, \xi) u(t, \xi)+L(0, \xi) v(t, \xi)) d \xi \\
V(t)=-\int_{0}^{1}(\bar{M}(1, \xi) u(t, \xi)+\bar{N}(1, \xi) v(t, \xi)) d \xi
\end{gathered}
$$

where $K, L$ and $\bar{M}, \bar{N}$ are defined by (52)-(55) and (44)(47), reaches its zero equilibrium in finite time $t_{F}$, where $t_{F}$ is given by (15). The zero equilibrium is exponentially stable in the $L^{2}$-sense.

Proof: Notice that evaluating (30) at $x=0$ yields (84) and evaluating (31) at $x=1$ yields (85). Since the kernels are invertible, there exists a unique operator $\mathcal{S}$ such that

$$
\left(\begin{array}{l}
u \\
v
\end{array}\right)=\mathcal{S}\left(\begin{array}{l}
\alpha \\
\beta
\end{array}\right)
$$

Applying Lemma 2 implies that $(\alpha, \beta)$ go to zero in finite time $t_{F}$, therefore $(u, v)$ converge to zero in finite time $t_{F}$ 


\section{Simulation RESUlts}

In this section we illustrate our results with simulations on a toy problem. The numerical values of the parameters are as follow.

$$
\lambda=0.5, \quad \mu=1, \quad \sigma^{+-}=0.5, \quad \sigma^{-+}=1
$$

Figure 3 pictures the $\mathcal{L}^{2}$-norm of the state $(u, v)$ in open loop and using the control law (84)-(85) presented in this paper. While the system in open loop is unstable (the $\mathcal{L}^{2}$-norm diverges), it converges in minimum time $t_{F}=\max \left\{\frac{1}{\lambda}, \frac{1}{\mu}\right\}=$ 2 when controller (84)-(85) is applied, as expected from Theorem 3.

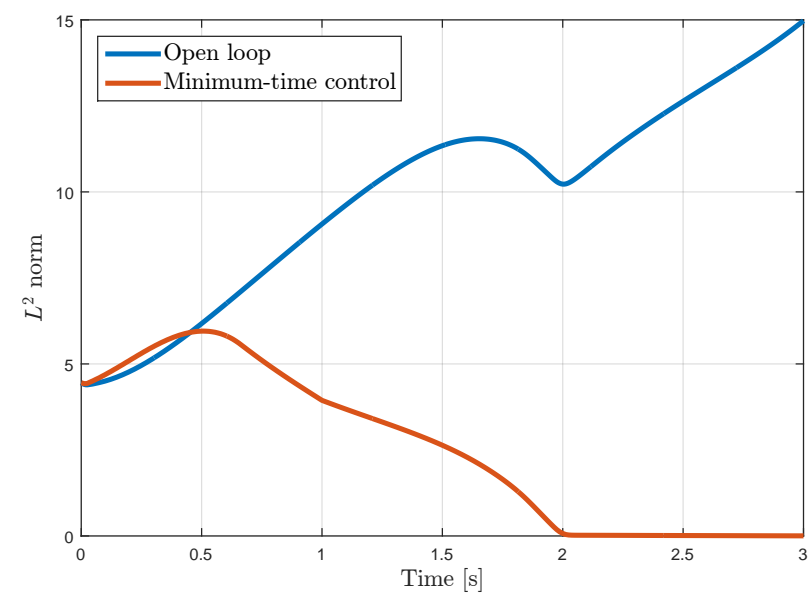

Fig. 3. Time evolution of the L2-norm in open loop and using the controler

\section{CONCLUDING REMARK}

Using the backstepping approach we have presented a stabilizating boundary feedback law for a system of firstorder hyperbolic linear PDEs controlled in both boundary. The zero equilibrium of the system is reached in minimum time $t_{F}$ which is the largest time between the two transport times in each direction.

This result is a first step towards completely bridging the gap between the theoretical results of [20] and explicit control design. By combining the presented approach with the result of [2], we believe it is possible to design a minimum-time stabilizing controller for general heterodirectional hyperbolic systems. The dual observer problem, crucial to envision application of this method on an industrial problem in a potential observer-controller structure, will also be the topic of future contributions.

\section{REFERENCES}

[1] Saurabh Amin, Falk M Hante, and Alexandre M Bayen, On stability of switched linear hyperbolic conservation laws with reflecting boundaries, Hybrid Systems: Computation and Control, Springer, 2008, pp. 602-605.

[2] Jean Auriol and Florent Di Meglio, Minimum time control of heterodirectional linear coupled hyperbolic pdes.

[3] G Bastin and JM Coron, Stability and boundary stabilization of 1-d hyperbolic systems, Preprint (2015).

[4] Haim Brezis, Functional analysis, sobolev spaces and partial differential equations, Springer Science \& Business Media, 2010.
[5] Federico Bribiesca-Argomedo and Miroslav Krstic, Backsteppingforwarding control and observation for hyperbolic pdes with fredholm integrals, Automatic Control, IEEE Transactions on 60 (2015), no. 8 , 2145-2160.

[6] Felipe Castillo Buenaventura, Emmanuel Witrant, Christophe Prieur, and Luc Dugard, Dynamic boundary stabilization of hyperbolic systems, 51st IEEE Conference on Decision and Control (CDC 2012), 2012, pp. n-c.

[7] Jean-Michel Coron, Control and nonlinearity, no. 136, American Mathematical Soc., 2009.

[8] Jean-Michel Coron, Georges Bastin, and Brigitte d'Andréa Novel, Dissipative boundary conditions for one-dimensional nonlinear hyperbolic systems, SIAM Journal on Control and Optimization 47 (2008), no. 3, 1460-1498.

[9] Jean-Michel Coron, Brigitte dAndréa Novel, and Georges Bastin, A lyapunov approach to control irrigation canals modeled by saintvenant equations, Proc. European Control Conference, Karlsruhe, 1999.

[10] Jean-Michel Coron, Long Hu, and Guillaume Olive, Stabilization and controllability of first-order integro-differential hyperbolic equations, arXiv preprint arXiv:1511.01078 (2015).

[11] Jean-Michel Coron, Rafael Vazquez, Miroslav Krstic, and Georges Bastin, Local exponential h^2 stabilization of a $2 \backslash$ times 2 quasilinear hyperbolic system using backstepping, SIAM Journal on Control and Optimization 51 (2013), no. 3, 2005-2035.

[12] Jonathan de Halleux, Christophe Prieur, J-M Coron, Brigitte d'Andréa Novel, and Georges Bastin, Boundary feedback control in networks of open channels, Automatica 39 (2003), no. 8, 1365-1376.

[13] Florent Di Meglio, Dynamics and control of slugging in oil production, Ph.D. thesis, École Nationale Supérieure des Mines de Paris, 2011.

[14] S Djordjevic, OH Bosgra, PMJ Van den Hof, and Dimitri Jeltsema, Boundary actuation structure of linearized two-phase flow, American Control Conference (ACC), 2010, IEEE, 2010, pp. 3759-3764.

[15] Stéphane Dudret, Karine Beauchard, Fouad Ammouri, and Pierre Rouchon, Stability and asymptotic observers of binary distillation processes described by nonlinear convection/diffusion models, American Control Conference (ACC), 2012, IEEE, 2012, pp. 3352-3358.

[16] James M Greenberg and Li Ta Tsien, The effect of boundary damping for the quasilinear wave equation, Journal of Differential Equations 52 (1984), no. 1, 66-75.

[17] Long Hu, Florent Di Meglio, Rafael Vazquez, and Miroslav Krstic, Control of homodirectional and general heterodirectional linear coupled hyperbolic pdes, arXiv preprint arXiv:1504.07491 (2015).

[18] Fritz John, Continuous dependence on data for solutions of partial differential equations with a prescribed bound, Communications on pure and applied mathematics 13 (1960), no. 4, 551-585.

[19] Daqian Li, Global classical solutions for quasilinear hyperbolic systems, vol. 32, John Wiley \& Sons, 1994.

[20] Tatsien Li and Bopeng Rao, Strong (weak) exact controllability and strong (weak) exact observability for quasilinear hyperbolic systems, Chinese Annals of Mathematics, Series B 31 (2010), no. 5, 723-742.

[21] Christophe Prieur and Frédéric Mazenc, Iss-lyapunov functions for time-varying hyperbolic systems of balance laws, Mathematics of Control, Signals, and Systems 24 (2012), no. 1-2, 111-134.

[22] Christophe Prieur, Joseph Winkin, and Georges Bastin, Robust boundary control of systems of conservation laws, Mathematics of Control, Signals, and Systems 20 (2008), no. 2, 173-197.

[23] Tie Hu Qin, Global smooth solutions of dissipative boundary-value problems for 1st order quasilinear hyperbolic systems, CHINESE ANNALS OF MATHEMATICS SERIES B 6 (1985), no. 3, 289-298.

[24] Valérie Dos Santos and Christophe Prieur, Boundary control of open channels with numerical and experimental validations, Control Systems Technology, IEEE Transactions on 16 (2008), no. 6, 1252-1264.

[25] Gerald Beresford Whitham, Linear and nonlinear waves, vol. 42, John Wiley \& Sons, 2011.

[26] Cheng-Zhong Xu and Gauthier Sallet, Exponential stability and transfer functions of processes governed by symmetric hyperbolic systems, ESAIM: Control, Optimisation and Calculus of Variations 7 (2002), $421-442$. 